\title{
Primary Malignant Melanoma of the Female Urethra
}

\author{
Sung Tae Cho, Hyeong Cheol Song, Byungchul Cho, Woo Seok Choi, Won Ki Lee, Yong Seong Lee, \\ Young Goo Lee, Ki Kyung Kim, Sung-Ho Park ${ }^{1}$, Jeong Won Kim ${ }^{2}$ \\ Departments of Urology, ${ }^{1}$ Obstetrics and Gynecology and ${ }^{2}$ Pathology, Hallym University College of Medicine, Seoul, Korea
}

\begin{abstract}
We report here on a rare case of primary malignant melanoma of the female urethra. A 69-year-old female presented at our hospital with a several month history of dysuria, poor stream, gross hematuria, intermittent blood spots, and a painful mass at the external urethral meatus. The physical examination revealed a soft, small, chestnut-sized lesion through the urethral orifice. The mass was tan colored, ulcerated, covered with necrotic tissue, and protruded from the external urethral meatus. The mass was removed by wide local excision under spinal anesthesia. The pathological diagnosis was malignant melanoma of the urethra. Computed tomography of the abdomen as well as a whole-body bone scan showed no evidence of metastasis. The patient has been free of disease for 6 months postoperatively. We discuss the clinicopathologic features and treatment of this tumor.
\end{abstract}

\section{Key Words: Female; Melanoma; Urethra}

This is an Open Access article distributed under the terms of the Creative Commons Attribution Non-Commercial License (http://creativecommons.org/licenses/by-nc/3.0) which permits unrestricted non-commercial use, distribution, and reproduction in any medium, provided the original work is properly cited.

\author{
Article History: \\ received 20 April, 2011 \\ accepted 13 May, 2011 \\ Corresponding Author: \\ Sung Tae Cho \\ Department of Urology, Hallym \\ University Kangnam Sacred Heart \\ Hospital, Hallym University College of \\ Medicine, 1 Singil-ro, \\ Yeongdeungpo-gu, Seoul 150-950, \\ Korea \\ TEL: $+82-2-829-5198$ \\ FAX: +82-2-846-5198 \\ E-mail: stcho@hallym.ac.kr
}

Primary malignant melanoma of the urethra in females is extremely rare and represents only $0.2 \%$ of all malignant melanomas [1]. To our knowledge, primary malignant melanoma involving the urethra in females has been reported in only three cases in the Korean literature [2]. Most malignant melanomas of the female urethra are located at the meatus or in the distal urethra [3]. The common presentations include bleeding, discharge, voiding dysfunction, or a mass [4]. Urethral melanomas are frequently misdiagnosed clinically, which leads to a delayed diagnosis, and patients with this tumor usually show a poor prognosis [4]. In this report, we present a case of melanoma originating from the urethra and we review the relevant medical literature.

\section{CASE REPORT}

A 69-year-old woman presented at our department with a several month history of dysuria, poor stream, gross hematuria, intermittent blood spots, and a painful mass at the external urethral meatus. She had no history of flank pain or passage of stones in the urine. She was postmenopausal and had no history of postmenopausal bleeding. She also had no history of hypertension, hyperlipidemia, diabetes, or pelvic surgery. The results of a chest X-ray and routine blood tests were normal at the presentation. The pelvic examination revealed a soft, chestnut-sized lesion protruding through the urethral orifice. The mass was tan colored, ulcerated, covered with necrotic tissue, and protruded from the external urethral meatus (Fig. 1). The findings of her speculum and vaginal examination of the cervix were unremarkable. The findings of her general physical examination were unremarkable. No organomegaly was found on an abdominal examination and no superficial lymph nodes were palpated. A cystourethroscopy was performed, which showed a polypoid, brownish growth involving only the external urethral meatus; there were no other abnormal findings in the bladder.

The mass was removed by wide local excision with the patient under spinal anesthesia. Gross examination of the resected specimen revealed a polypoid solid mass (4.0x3.0x $2.0 \mathrm{~cm}$ ) with a dark-brown cut surface. The mass was covered with pinkish mucosa with surface ulceration. Microscopic examination demonstrated large epithelioid tumor cells with dusty brown melanin pigments and frequent mitotic figures (Fig. 2). Immunohistochemical staining showed that the malignant cells were immunoreactive for HMB45 (Fig. 3A) and S-100 protein (Fig. 3B), which are sensitive 
markers for melanocyte differentiation. A macroscopically clean surgical margin was obtained. However, histology performed later revealed a microscopic positive margin. On the basis of these findings, the patient was diagnosed as having primary malignant melanoma of the urethra, stage T4bNxM0.

Abdominal computed tomography detected no lymphatic nodal swelling or distant metastases. The whole-body bone scan also revealed no evidence of metastasis. There was no evidence of any other primary tumor. We recommended additional radical urethrectomy to obtain a wide enough surgical margin. However, the patient refused additional surgery owing to her anxiety over losing a functional organ. Although radiotherapy and chemotherapy

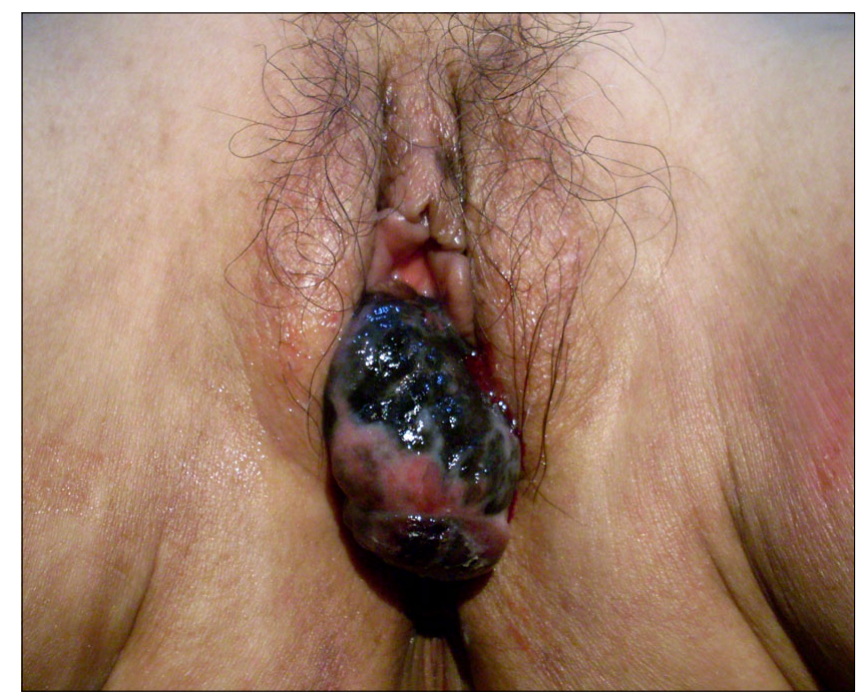

FIG. 1. The appearance of the urethral malignant melanoma at presentation. There is a polypoid, tan colored, ulcerated mass of the external urethral meatus. were advised, she refused any further treatment. The patient is currently under close follow-up. Clinical and radiological evaluations take place every 3 months. The patient has experienced no progression in disease, including systemic metastasis, for more than 6 months as assessed by abdominal computed tomography and cystourethroscopy.

\section{DISCUSSION}

Malignant melanoma is one of the rarest tumors of the female urethra and usually originates from the distal urethra [1]. It accounts for $0.2 \%$ of all malignant melanomas and $4 \%$ of all urethral cancers [3]. The first case of urethral melanoma was reported by Tyrell and Reed more than 100 years ago [4,5]. A few additional cases have been reported

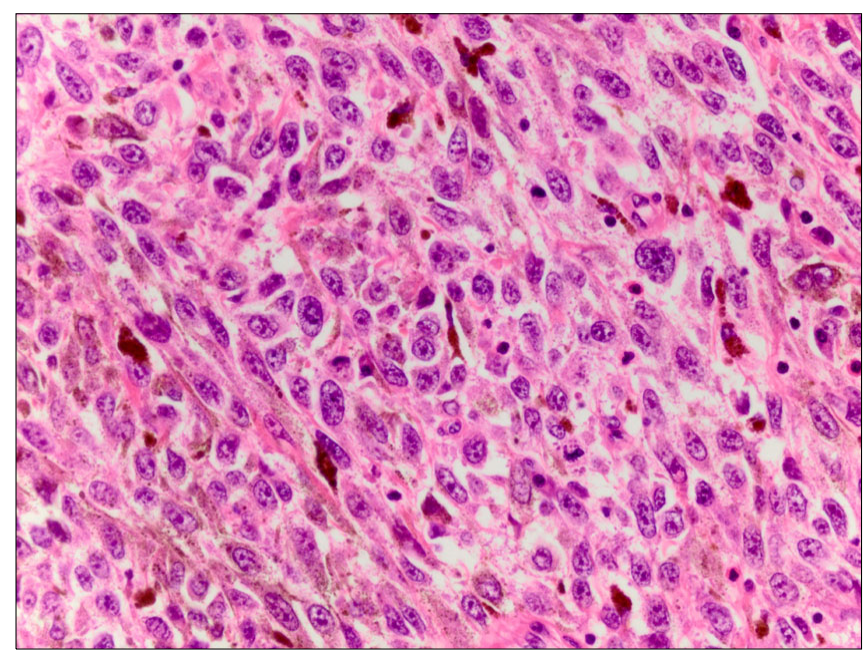

Fig. 2. Light microscopic findings of the surgical specimen. The large epithelioid tumor cells have dusty brown melanin pigments and frequent mitotic figures are seen (H\&E, x400).
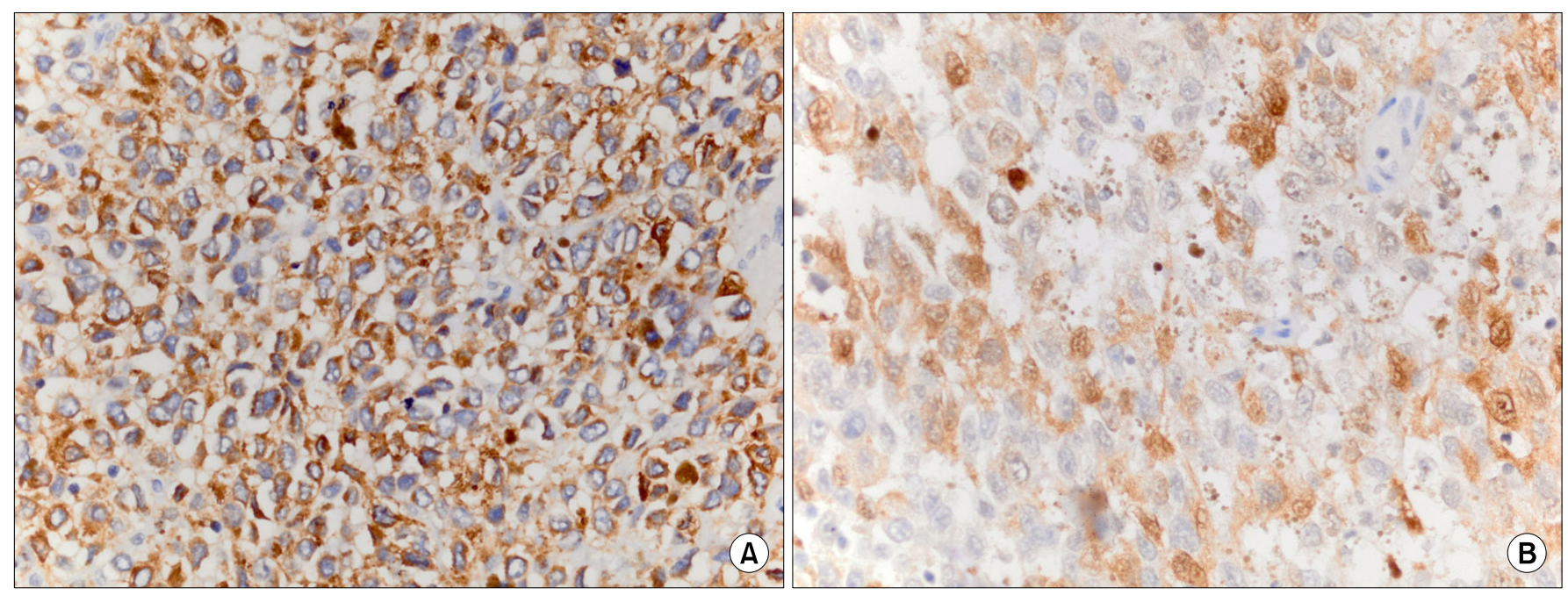

Fig. 3. The immunohistochemical staining findings of the surgical specimen. The tumor cells were immunoreactive for HMB-45 (A) and S-100 protein (B). 
since that time. Only five cases of urethral melanoma have been reported in the Korean literature, three in women and two in men [2].

Urethral malignant melanoma is three times as common in women as in men, like other malignant urethral tumors, and it is more frequent in the Caucasian population. Most cases are diagnosed after the fifth decade but some cases occur in younger patients [6,7]. The presenting symptoms include vaginal bleeding, mass, hematuria, and dysuria with a decreased urinary stream [8].

Microscopic examination of urethral malignant melanomas demonstrates a wide histologic spectrum with patterns that include diffuse, nested, fascicular, and storiform growths of pleomorphic cells [4]. This variability has made immunohistochemical markers for melanocyte differentiation a central part of making an accurate diagnosis. The most frequently used melanocytic markers in clinical practice are S-100 protein and HMB-45. Monoclonal antibody to S-100 protein is a sensitive marker that reacts with more than $90 \%$ of melanomas [9]. Although HMB-45 is quite specific for melanocytic neoplasms, it is less sensitive than S-100 protein for identifying melanoma [9]. In our case, as a result of this high sensitivity, the strong immunoreactivity to $\mathrm{S}-100$ protein resulted in a diagnosis of malignant melanoma. Subsequent testing with HMB-45, a marker with higher specificity for melanocytic neoplasms, was conclusively positive and thus provided a definitive diagnosis.

The urethral malignant melanoma has a worse prognosis than a malignant melanoma of a cutaneous origin, partly because of the frequent finding of a vertical growth phase and lymph node metastasis at the time of initial diagnosis and partly because the location often results significant delays in making the diagnosis [5]. Clinically, urethral malignant melanomas are frequently polypoid and are usually mistaken for other malignant diseases or even benign lesions, including urethral polyps, caruncle, mucosal prolapse, or chancre [4]. A few cases of urethral malignant melanoma that were initially suspected to be urethral caruncle have been reported [10].

Primary malignant melanoma of the female urethra tends to metastasize at an early stage to adjacent areas, the regional lymph nodes, and occasionally distant sites by the hematogenous route. Therefore, in the case of malignant melanoma in the urethra, the treatment of choice is early extensive excision to control the local disease [2].

The treatment recommendations primarily depend on the tumor location and the clinical stage. The treatment varies from local excision with or without the addition of radiation therapy to extensive surgery, including cystourethrectomy, vaginectomy, vulvectomy, and lymph node dissection [7]. Local excision, which should lead to good functional results, may be sufficient for relatively small, superficial, distal urethral tumors. However, local excision may not be adequate for good local control. A more aggressive approach is needed for more proximal and advanced urethral tumors. In the present case, we performed wide local excision. In addition, we recommended additional radical urethrectomy with bladder preservation to obtain a wide enough surgical margin and to prevent the progression of the disease. However, when total urethrectomy is done, patients must undergo urinary diversion, which requires an external appliance, and thus the patient's quality of life suffers. Ultimately, our patient refused additional surgery because of anxiety over losing a functional organ.

The optimum treatment for urethral melanomas in women has not yet been defined. Several different surgical approaches were used in the cases with long-term survival that were described in the literature, but no definitive conclusions can be drawn because of the limited number of patients and the lack of information about the depth of invasion in some of these cases [5]. Radical surgical resection with postoperative adjuvant chemotherapy or immunotherapy is recommended, but little clinical experience exists to support such adjuvant treatment. Occasional long-term survivors have been noted after radiation therapy, and this suggests the potential role of postoperative radiation therapy to the tumor bed or to the regional lymphatics in high-risk patients [8].

\section{CONFLICTS OF INTEREST}

The authors have nothing to disclose.

\section{REFERENCES}

1. Akbas A, Akman T, Erdem MR, Antar B, Kilicarslan I, Onol SY. Female urethral malignant melanoma with vesical invasion: a case report. Kaohsiung J Med Sci 2010;26:96-8.

2. Lee JS, Kang TW, Kwon DD, Park KS, Ryu SB, Park YI. Primary Periurethral Malignant Melanoma. Korean J Urol 2005;46:203-5.

3. Kim CJ, Pak K, Hamaguchi A, Ishida A, Arai Y, Konishi T, et al. Primary malignant melanoma of the female urethra. Cancer 1993;71:448-51.

4. Filipkowski LA, Barker MA, Karram MM. Primary genitourinary melanoma presenting as voiding dysfunction. Int Urogynecol J Pelvic Floor Dysfunct 2009;20:1141-3.

5. Sugiyama VE, Chan JK, Kapp DS. Management of melanomas of the female genital tract. Curr Opin Oncol 2008;20:565-9.

6. Gupta R, Bhatti SS, Dinda AK, Singh MK. Primary melanoma of the urethra: a rare neoplasm of the urinary tract. Int Urol Nephrol 2007;39:833-6.

7. Oliva E, Quinn TR, Amin MB, Eble JN, Epstein JI, Srigley JR, et al. Primary malignant melanoma of the urethra: a clinicopathologic analysis of 15 cases. Am J Surg Pathol 2000;24:785-96.

8. Piedimonte A, Aquinati A, Scalise A, Bertani A. Occult primary melanoma of the urethra: a long-term survival case. Plast Reconstr Surg 2004;114:618-9.

9. Sheffield MV, Yee H, Dorvault CC, Weilbaecher KN, Eltoum IA, Siegal GP, et al. Comparison of five antibodies as markers in the diagnosis of melanoma in cytologic preparations. Am J Clin Pathol $2002 ; 118: 930-6$.

10. Nakamoto T, Inoue Y, Ueki T, Niimi N, Iwasaki Y. Primary amelanotic malignant melanoma of the female urethra. Int J Urol 2007;14:153-5. 